\title{
XIV.
}

Aus dem pharmakologischen Institut zu Heidelberg.

\section{Ueber Diurese.}

\section{Mittheilung:}

Die Beziehungen des Ureterendruckes zur Diurese.

von

R. Gottlieb und R. Magnus.

(Mit 1 Curve.)

Als ein weiteres Mittel, um über die Beziehungen zwischen den Cireulationsverhältnissen in der Niere und der Harnabsonderung Aufscbluss zu erlangen, diente seit den Untersuchungen Max Hermann's 1) das Studium der Druckveränderungen, welche ein endständig mit dem Ureter verbundenes Manometer darbietet. Da eine systematische Durcharbeitung dieser Druekänderungen bei der Diurese bislang nicht vorliegt, so soll im Folgenden uber derartige Versuchsreihen berichtet werden. Vorerst müssen wir aber kurz auf die physiologische Bedeutung des Ureterendruckes eingehen; denn die Diureseversuche ergaben auch einige nene Anhaltspunkte zur Beurtheilung der Frage, ob der Ureterendruck als Secretionsdruck der Niere aufgefasst werden darf.

Auch zu diesen Experimenten wurden vorzugsweise Kaninchen benutzt, denen in Chloral- oder Urethannarkose Canülen in beide Ureteren eingeführt wurden. Nachdem festgestellt war, dass auf beiden Seiten der Harn gut abtropfte, wurde der eine Ureter mit einem kleinen $\mathrm{Hg}$-Manometer verbunden, der andere blieb frei. So liess sich an der einen Niere der Ureterendruck, an der anderen die freie Harnabsonderung beobachten. Gleichzeitig wurde der Carotidendruck registrirt. Nach Schluss des Versuches wurde dann das Manometer wieder abgenommen, der Harn tropfte aus dem freigewordenen

1) Max Hermann, Sitzungsber. der Wiener Academie. Bd. XXXVI. 1859 and Bd. XLV. 1861 . 
Ureter ab, und es wurde auf diese Weise constatirt, dass der Weg von der Niere zum Manometer nicht etwa inzwischen durch Gerinnsel verlegt worden war.

Schon Hermann hatte beobachtet, dass unter normalen Verhältnissen der Druck im Ureter höchstens bis $60 \mathrm{~mm}$ steigt. Auch Heidenhain fand bei einem Aortendruck von 100-105 mm als böchsten Werth für den Ureterendruck $64 \mathrm{~mm}$, bei geringerem Blutdruck entsprechend niedrigere Werthe. Wir haben in unseren Versuchen Normalwerthe von $66 \mathrm{~mm}$ (Blutdruck 105), $66 \mathrm{~mm}$ (Blutdruck 93) und $62 \mathrm{~mm}$ (Blutdruck $103 \mathrm{~mm}$ ) beobachtet. Andererseits sahen wir auch Fälle, wo trotz hohen Blutdruckes der Ureterendruck erheblich niedriger war: $40 \mathrm{~mm}$ bei einem Blutdruck von 110 und $33 \mathrm{~mm}$ bei einem Blutdruck von $106 \mathrm{~mm}$.

Raschen Schwankungen des arteriellen Blutdruckes pflegt aueh das Ureterenmanometer zu folgen. So sahen wir z. B. nach Injection von $0,02 \mathrm{~g}$ Coffeïn natr. benz. den Blutdruck von $105 \mathrm{~mm}$ rapide auf $50 \mathrm{~mm}$ sinken und gleichzeitig den Ureterendruck von 66 auf $36 \mathrm{~mm}$ herabgehen, dann hob sich der Blutdruck wieder auf 110 und das Manometer auf $60 \mathrm{~mm}$.

Etwaige Contractionen des Ureters scheinen so gut wie gar keinen Einfluss auf den Stand des Manometers auszuiben. Ist das Maximum des Ureterendruckes erreicht, so ist der Ureter ausgeweitet, die Flüssigkeit im Nierenbecken und im Manometer steht in directem continuirlichem Zusammenhang ${ }^{1)}$ und irgendwelche Schwankungen, welche auf Uretercontractionen zu beziehen wären, haben wir nicht beobachtet. Bei Kaninchen schwankt der Quecksilbermeniseus überhaupt lkaum, es sind nur manchmal dem Pulse synchrone, ganz minimale Sehwankungen zu sehen, bei Hunden sind solehe jedoch deutlich ausgesprochen. Hier fallen sie ganz genau wit den Wellen des arteriellen Blutdruckes zusammen, sind also von diesem abhängig. Die pulsatorischen Druckänderungen in den Nierengefässen theilen sich demnach durch die trennenden Membranen dem Inbalt der Harncanälchen mit. Grössere Schwankungen des Ureterenmanometers treten auf, wenn das Thier sich wirft und Contractionen mit den Bauchmuskeln ausführt. Man muss natürlich bei derartigen Unterbrechungen abwarten, bis das Thier wieder ruhig wird und das Manometer den alten Stand eingenommen hat.

1) In einigen Versuchen wurde das Manometer mit einer Canüle verbunden, welche direct ins Nierenbecken eingeführt war. Ein Unterschied ergab sich hierbei nicht. 
Schliesst man das Manometer an den Ureter an, so steigt das Quecksilber Anfangs rasch, später immer langsamer, bis es seinen höchsten Stand erreicht, über den es nicht hinausgeht. Diese Form der Anstiegscurve bängt nach Hermann's Untersuchungen davon $a b$, dass die Geschwindigkeit, mit der der Harn aus der Niere in den Ureter tritt, mit wachsendem Widerstand stark abnimmt. Der höchste Stand des Ureterenmanometers giebt den Punkt an, bei welchem dieser Abfluss des Harnes ganz sistirt wird.

Hier setzt nun die Controverse tuber die Bedeutung des Ureterendruckes ein. Lud wi ga nahm an, dass der höchste Stand des Ureterenmanometers den Druck angebe, welcher nöthig sei, um die weitere Filtration von Harn in den Glomerulis hintanzuhalten, also den Druck, der dem Glomerulusdruck das Gleichgewicht halte. Es soilte also bei dem höchsten Stande des Manometers gerade die Harnabsonderung aufhören und das Manometer somit den Secretionsdruck der Niere anzeigen. Hiergegen wandte Heidenhain ein, dass die Secretion im Glomerulus sehr wohl weiter gehen könne, auch wenn das Manometer unverändert auf seinem höchsten Punkte verharrt, wenn nur gleichzeitig eine Rúckresorption von Harnflüssigkeit in den Tubulis stattfände. Nach Heidenhain giebt demnach der Ureterendruck nicht den Secretionsdruck der Niere an, sondern den Druck, bei dem sich Secretion und Resorption gerade das Gleichgewicht halten.

Zur Entscheidung dieser Frage können wir folgende, bei Anwendung von Diureticis gewonnene Beobachtungen heranziehen,

In mehreren Versuchen erreichte der Ureterendruck (nach Diureticis) eine gewisse Steigerung gegen die Norm, um dann wieder abzusinken. Nimmt man an, dass die Menge des in den Harncanälchen vorhandenen Harnes nicht abnimmt (Ludwig), so kann dieses Absinken nur bedingt sein durch gleichzeitiges Absinken des von aussen auf die Harncanälchen wirkenden Druckes, d. h. des Blutdruckes; gerade so, wie in einem mit einem Manometer verbundenen Gummiball der Druck sinkt, wenn man aufhört, den Ball mit der Hand zu comprimiren. Nimmt man dagegen mit Heiden$\mathrm{h}$ a in eine Rückresorption in den Harncanälchen an, so kann das Absinken des Ureterendruckes auch noch durch ein zweites Moment, dureh rermehrte Aufsangung des Harnes (bezw. fortdauernde Aufsaugung bei abnehmender Absonderung) bedingt sein.

Ist also die $\mathrm{Ludw}$ ig'sohe Lehre richtig, so muss jedes Sinken des Ureterendruckes ron einem Sinken des Blutdruckes begleitet sein. Besteht dagegen die Ansicht Heidenhain's zu Recht, so 
muss der Ureterendruck auch bei gleichbleibendem, ja steigendem Blutdruck absinken können. In der That zeigte sich in unseren Diureseversuchen diese letztere Eventualität verwirklicht:

Versueh VI: Absinkende NaCl-Diurese. Kaninchen, $1830 \mathrm{~g}$. 0,6 pro Kilo Chloralhydrat.

\begin{tabular}{lcc} 
& Blutdruck & Ureterendruck \\
$6 \mathrm{h.} 23 \mathrm{~m}$. & $122 \mathrm{~mm}$ & $62 \mathrm{~mm}$ \\
$6 \mathrm{~h} .34 \mathrm{~m}$. & $120=$ & $42=$ \\
\cline { 2 - 2 } & $-2 \mathrm{~mm}$ & $-20 \mathrm{~mm}$
\end{tabular}

Versuch VIII: Steigende Coffeïndiurese. Kaninchen, 1820 g. 0,6 pro Kilogramm Chloralhydrat.

\begin{tabular}{|c|c|c|}
\hline \multirow{4}{*}{$\begin{array}{l}5 \mathrm{~h} .22 \mathrm{~m} . \\
5 \mathrm{~h} .36 \mathrm{~m} . \\
\text { Differenz: }\end{array}$} & Blutdruck & Ureterendruck \\
\hline & $105 \mathrm{~mm}$ & $66 \mathrm{~mm}$ \\
\hline & $110=$ & $52=$ \\
\hline & $+5 \mathrm{~mm}$ & $-14 m$ \\
\hline
\end{tabular}

Es ist also in Versuch VI der Ureterendruck bei gleichbleihendem Blutdruck um $1 / 3$ seines Werthes gefallen, im Versuch VIII ist der Blutdruck sogar gestiegen, während der Ureterendruck sank. Diese Ergebnisse lassen einen anderen Schluss kaum zu, als dass der Ureterendruck nicht den Secretionsdruck der Niere angiebt, sondern nur den Druck, bei dem Secretion und Rückresorption sich genau das Gleichgewicht halten.

Diese Rückresorption aus den Harnwegen bei gehindertem Abfluss aus dem Ureter berechtigt uns jedoch durchaus nicht zu der Folgerung, dass eine solche Wiederaufsaugung auch unter normalen Bedingungen, bei offenem Abfluss des Harnes nach aussen, eine Rolle spiele. Wir baben es hier offenbar mit abnormen Verhältnissen zu thun und man sieht deutlich, dass die Niere während des Versuches allmählich wasserreicher, durchtränkter wird. Welchen Grad dieser pathologische Flüssigkeitsdurchtritt erreichen kann, davon kann man sich ein Bild machen, wenn man einem Thier nach Unterbindung der Ureteren längere Zeit physiologische Kochsalzlösung intravenös infundirt. Dann findet man nachher die Niere und den Ureter eingebettet in eine voluminöse, starre, glasige Snlze, aus der beim Ausscheiden reichlich Flüssigkeit abfliesst, ein enormes perirenales Oedem. 1)

Im Anschluss an C. Lud $\mathrm{d}$ ig hat dagegen Starling in jüngster Zeit eine andere Auffassung des Ureterendruckes zu begründen ver-

1) Vgl. Magnus, Ueber die Entstehung der Hautödeme u. s. w. Archiv f. experiment. Patbol. u. Pharmakol. Bd. XLII. S. 274. 1899. 
sucht. Ustimowitseh 1) zeigte bekanntlich, dass bei einem arteriellen Blutdruck von $40-50 \mathrm{~mm} \mathrm{Hg}$ die Harnsecretion aufhört. Unter günstigen Bedingungen sah Grützner noch bei einem Blutdruck von $30 \mathrm{~mm}$ den Harn fliessen. Stets aber war ein gewisses Minimum von Blutdruck, von "vis a tergo" nöthig, damit die Niere Harn lieferte.

Starling ${ }^{2}$ ) hat nun versucht, diese Beobachtungen mit für seine Anschauungen zu verwenden, dass im Glomerulus ein Filtrationsprocess vor sich gehe. Er sieht im Ureterendruck ein Derivat des Blutdruckes in den Glomerulis der Niere und meint, dass er immer etwa um $40 \mathrm{~mm}$ unter dem Blutdruck bleibe; diese $40 \mathrm{~mm} \mathrm{Hg}$ seien gleich dem osmotisehen Drucke des Eiweisses. Werde nun Serum minus Eiweiss unter dem Glomerulusdruck a durch den Glomerulus filtrirt, so müsse der Druck in den Harncanälehen $a-b$ betragen, wenn b der osmotische Druck des Eiweisses sei. Der Ureterendruck sei also gleich dem Blutdruck minus dem osmotischen Drucke des Eiweisses. Auf diese Weise erkläre es sioh, dass der Ureterendruck immer um ea. $40 \mathrm{~mm}$ unter dem Blutdrucke bleibe und dass bei einem Blutdrucke von 30-40 mm die Secretion ganz versiegt.

Abgesehen davon, dass wir aus den oben angeführten Gründen im Ureterendruck nicht den Filtrationsdruck in den Glomerulis sehen dürfen, können unsere Versuche auch weiterhin noch zur Kritik dieser Anschauungen dienen. Es stellte sich nämlich heraus, dass die Differenz zwischen Blutdruck und Ureterendruck durchaus nicht immer dem Starling'schen Werthe entspricht:

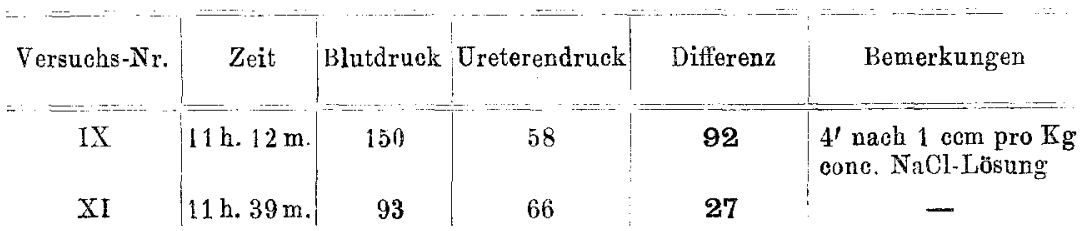

In Versuch IX bestand Kochsalzdiurese, demnach Blutverdünnung, und der osmotische Druck des Bluteiweisses hätte gesunken sein müssen: trotzdem eine Differenz von $92 \mathrm{~mm}$. In Versuch XI handelt es sich um eine Normalsecretion: trotzdem nur eine Differenz von $27 \mathrm{~mm}$. Auch hieraus ergiebt sich, dass die Beziehungen zwisehen Blutdruck und Ureterendruek unmöglich so einfach sein können, wie dies Starling annimmt.

1) Ustimowitsch, Leipziger Arbeiten. 1870. S. 198.

2) Starling, Journ. of physiol. Vol. XXIV. S. 317. 1899. 
Den interessantesten Aufschluss in dieser Frage gab uns ein Experiment, das in Curvenform ausführlich mitgetheilt werden soll. Wir wollten den Versuch machen, ob es nicht doch gelänge, den Ureterendruck über den Blutdruck zu steigern, und verfuhren zu dem Zweeke so, dass wir bei einem Kaninchen dureh continuirliche intravenöse Inffusion von warmer 0,9 procent. NaCl-Lösung starke Nierenthätigkeit einleiteten, während dieser starken Diurese aber zugleich den Blutdruck allmählich herabsetzten durch wiederholte intravenöse Einspritzung einer 4 procent. Chloralhydratlösung. Es

Versuch XII.

Kaninchen, $2450 \mathrm{~g}$. Trachealcanüle. Künstliche Athmung von der 28. Minute an. Manometer links. Secretion rechts.

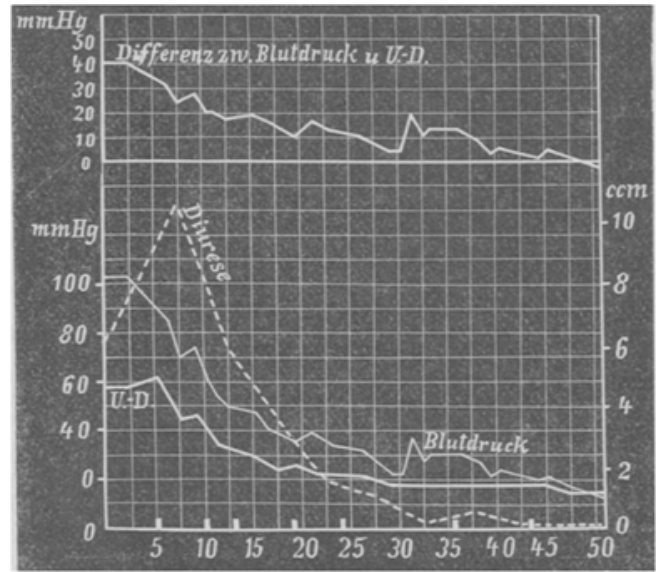

Injection von 0,08 Chloralhydrat pro Kilogramm (4 Proc.).

ist uns nun allerdings nicht gelungen, den Carotidendruck unter den Ureterendruok sinken zu sehen, aber die Resultate waren auch ohnehin interessant genug.

Man sieht, wie in Folge der Chloralhydratgabe der Blutdruck allmählich von $103 \mathrm{~mm}$ sich senkt, um schliesslich bei $13 \mathrm{~mm} \mathrm{zu}$ enden. Die Harnsecretion, welche Anfangs den Werth von über $10 \mathrm{ccm}$ pro 5 Minuten und 1 Kilo Thier erreichte, sinkt ebenfalls continuirlich ab. Von der 17. Minute ab bleibt der Blutdruck dauernd unter $40 \mathrm{~mm}$. Trotzdem wurden von dem $21 / 2 \mathrm{~kg}$ schweren Thiere nachher noch $18 \mathrm{com}$ Harn geliefert. Von der 37. Minute bleibt der Blutdruek unter $30 \mathrm{~mm}$, trotzdem erfolgt noch Bildung von $0,3 \mathrm{com}$ Harn. Ja! als der arterielle Blutdruck zwischen 16 und $13 \mathrm{~mm}$ schwankte, tropfte noch deutlich ein neugebildeter Tropfen aus der 
Ureterencanüle ab. Der Harn blieb während dieses Versuches eiweissfrei.

Während der Blutdruck continuirlich absinkt, geht auch der Ureterendruck allmählich herab, aber langsamer; er kommt also dem Blutdruck immer näher. Von der Höhe von $60 \mathrm{~mm}$ geht er auf $14 \mathrm{~mm}$ zum Schluss herab. Bei einem Uretereudruck von $25-18 \mathrm{~mm}$ ist noch kräftige Harnseeretion der anderen Niere vorhanden. Wie sich die Differenz zwischen Blutdruck und Ureterendruck ändert, ersieht man aus der obersten Curve. Diese Differenz ist Anfangs $40 \mathrm{~mm}$, nimmt dann aber continuirlich ab, und von der 15. Minute ist sie dauernd geringer als $20 \mathrm{~mm}$, ja gegen Schluss des Versuches näbert sie sich immer mehr dem Nullwerth. Bei einer mittleren Differenz von $6 \mathrm{~mm} \mathrm{Hg}$ wird in 5 Minuten noch $0,3 \mathrm{ccm}$ secernirt. Der vorletzte Tropfen fliesst, während Ureterendruck und Blutdruck nur noch $2 \mathrm{~mm}$ von einander entfernt sind. Ja, am Schluss ist der Blutdruck nur $13 \mathrm{~mm}$, der Ureterendruck $14 \mathrm{~mm}$.

Es zeigt sich also, dass unter gewissen Bedingungen die Harnsecretion auch bei sehr niedrigem Blutdruck noch fortdauern, ja dass bei 13-16 mm Carotidendruck noch sichere Harnbildung stattfinden kann. Und weiter ergiebt sich, dass es möglich ist, den Ureterendruck dem arteriellen Blutdruck sehr stark zu nähern, dass ihre Differenz nur noch $2 \mathrm{~mm} \mathrm{Hg}$ beträgt und dass auch bei so geringem Abstand der Druckwerthe noch Harn producirt werden kann. Berücksichtigt man dabei, dass jedenfalls der Blutdruck in den Nierencapillaren unter dem Carotidendruck liegt, so kann man sagen, dass auch bei fortdauernder Harnsecretion Blutdruck und Ureterendruck annähernd gleiche Werthe erreichen können.

Der angeführte Versuch ist wohl ungemein geeignet, die ausschlaggebende Bedeutung der veränderten Blutzusammensetzung zu demonstriren, auf welche wir in diesen Mittheilungen immer wieder hinweisen müssen. Die Gegenwart der harnfäbigen Stoffe zwingt die Niere zur Thätigkeit, obgleich der "Filtrationsdruck" in den Glomerulis auf ein Minimum herabgedruiekt ist. Unser Versuch stellt in diesem Sinne einen extremen Fall der schon von Ustimowitseh beobachteten relativen Unabhängigkeit der Harnabsonderung vom Blutdruck dar. Die Beobachtung von Ustimowitseh, dass Kochsalz- und Harnstoffinjection auch nach Rückenmarksdurchsehneidung noch Diurese hervorrufen kann, hat sehon Ludwig zu einem Zusatz zur Druckhypothese gezwungen (a. a. 0. S. 217), „dass die Wirksamkeit des Druckes in einer Abhängigkeit stebe von dem Gehalte des Blutes an harnfähigen Stoffen und zwar in der Art; dass eine 
gegebene Differenz der Spannungen erst bei einem bestimmten Gehalte des Blutes an Harnbestandtheilen wirkungsfähig: werden, bezw. um so mehr Harn liefern könne, je grösser die Anhäufung der Harnbestandtheile im Blute geworden sei". Nur suchte Ludwig den Grund dieser Abhängigkeit der Diurese von den harnfähigen Stoffen in einer passiven Veränderung der Durchgängigkeit der den Glomerulus umgebenden Bäute durch die Vermehrung der Harnbestandtheile im Blute, oder in einer weiteren secundären Veränderung "des durch den Glomerulus abgesonderten Harnes auf seinem Wege durch die Harneanälchen", während Heidenhain in den harnfähigen Substanzen den Reiz zur specifischen Zellthätigkeit erblickt.

Da in unserem Falle die zur Verfügung stehende Druckdifferenz in den Glomerulis jedenfalls eine ganz minimale war und dennoch Harnabsonderung erfolgte, so tritt die Bedeutung der harnfähigen Stoffe gegenï̈ber den Druckverhältnissen im Glomerulus auf das deutlichste hervor.

Zusammenfassend lässt sich sagen, dass die Differenz zwischen Blutdruck und Ureterendruck keine constante ist, dass Werthe über $90 \mathrm{~mm}$ Ureterendruck vorkommen, dass bei einem sehr niedrigen Werthe noch starke Harnsecretion stattfinden kann und dass es Fälle giebt, wo bei fast gleichem Blut- und Ureterendruck noch ein $\mathrm{Ab}$ tropfen vom Urin stattfindet. Es kann demnach der osmotisehe Druck des Eiweisses im Sinne Starling's für die Differenz zwischen Blutund Ureterendruck nicht verantwortlich gemacht werden.

Ueber die Frage, wie sich der Ureterendruck bei der Diurese ändert, geben nachstehende Versuche Aufschluss. Diese Versuchsreihe war unternommen in der Hoffnung, vielleicht irgendwelche charakteristisehen Unterschiede zwischen Coffeïn- und Salzdiurese zu finden. Diese Erwartung hat sich aber nicht erfüllt.

Wartet man, bis sich das Manometer fest eingestellt hat und leitet dann Diurese ein, so beobachtet man am Ureterendruck folgendes :

\section{a. Kochsalzdiurese.}

In einer gewissen Zahl von Fällen steigt nach intravenöser Injection von starken $(5,10$ und 35 procent.) NaCl-Lösungen der Ureterendruck rasch an und erreicht einen hohen Werth:

Versuch IV ron 33,5 auf $52 \mathrm{~mm} \mathrm{Hg}(2 \mathrm{ecm}$ conc. $\mathrm{NaCl})$.

VI $=33=62==(0,5$ cem conc. $\mathrm{NaCl})$, während der Blutdrnck von 108 anf $122 \mathrm{~mm}$ steigt

Versuch III von 40 auf $60 \mathrm{~mm} \mathrm{Hg}(38 \mathrm{ccm} 5$ procent. $\mathrm{NaCl})$, während der Blutdruck von 110 auf $102 \mathrm{~mm}$ sinkt. 
Diese Drucksteigerung geht sehr sehnell vor sich, manchmal schiesst das Quecksilber geradezu in die Höhe, um dann seinen definitiven hohen Stand mit abnehmender Geschwindigkeit zu erreichen. Das Manometer bleibt dann einige Zeit auf der Höhe, um mit absinkender Diurese ebenfalls wieder langsam zu fallen (vgl. S.250). Dabei kann die Diurese noch andauern, während der Ureterendruck sohon zur Norm zurückgekehrt ist.

Während sich in den eben geschilderten Fällen der Ureterendruck rasch bei der Kochsalzdiurese von verhältnissmässig niederen Werthen zu grossen Höhen hob, beobachtet man in anderen Versuchen, trotzdem eine starke Kochsalzdiurese auftritt, kein Ansteigen des Ureterenmanometers. Dieses ist meist in den Fällen zu erwarten, in denen schon während der Normalsecretion ein hoher Ureterendruck beobachtet wurde.

Versuch XI. Kaninchen, $1850 \mathrm{~g}$. 0,6 pro $\mathrm{Kg}$ Chloralhydrat. Vor der NaCl-Injection:

Ureterendruck 58-62 mm. Harnmenge 0,5 ccm in 10 Minuten.

Nach Injection von $2 \mathrm{ecm}$ conc. $\mathrm{NaCl}$ :

Ureterendruck $62-58 \mathrm{~mm}$. Harnmenge $11,0 \mathrm{ecm}$ in 10 Minuten.

(Dabei war die Canüle ganz frei, denn nach der Abnahme des Manometers erfolgte kräftiger Harnabfluss.)

Man kann also im Allgemeinen die Regel aufstellen, dass bei niederem Normaldruck durch die Diurese ein deutliehes Ansteigen des Ureterendruckes bewirkt wird, während dieses bei hohem Normaldruck meist nicht zu erwarten ist.

b. Wasserdiurese.

Bei einem Hund von 11//2 kg lief in $1 / 2$ Stunde ca. 110,9 proc. Kochsalzlösung ein. Dabei stieg die Harnabsonderung auf 50,0 cem in 10 Minuten. Der Ureterendruck hob sich ron $52 \mathrm{~mm}$ in $15 \mathrm{Mi}$ nuten auf $76 \mathrm{~mm}$ und hielt sich bis zum Schluss des Einlaufes auf dieser Höhe. Dabei war der Blutdruck vor dem Einlauf $145 \mathrm{~mm}$, am Schlusse $140 \mathrm{~mm} \mathrm{Hg}$.

\section{c. Coffeïndiurese.}

Für die Coffeïndiurese gilt im Allgemeinen das Gleiche, wie für die Kochsalzdiurese. In einer Reihe von Fällen gelingt es, eine deutliche Steigerung des Ureterendruckes zu erzielen:

Versuch $V$ durch 0,04 Coff. natr. benz. von 55 auf $84 \mathrm{~mm} \mathrm{Hg}$

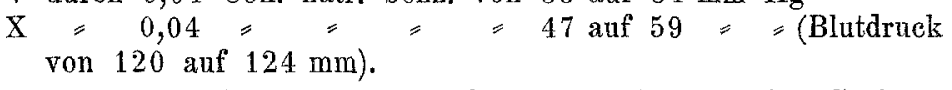

Der Verlauf ist dabei so, dass bei der Injection des Coffeïns 
meist eine schnelle, aber kurzdauernde Blutdrucksenkung erfolgt, welche ein gleichzeitiges, kurzdauerndes Absinken des Ureterendruekes zur Folge hat, dass dann aber der Blutdruck alsbald zur Norm zurïckgeht, während sich das Ureterenmanometer auf seinen höheren Werth einstellt. Mit dem Absinken der Diurese geht auch der Ureterendruck wieder zurück, ohne dass jedoch ein genauer Parallelismus zwisehen beiden bestände.

In einer anderen Reihe von Fällen tritt deutliche Diurese, aber kein Ansteigen des Ureterendruckes ein:

Versuch VI. Kaninchen, 1830 g. 0,6 pro $\mathrm{Kg}$ Chloralhydrat.

\begin{tabular}{|c|c|c|c|}
\hline Zeit & Ureterendruck & Blutdruck & Harnmenge in $10 \mathrm{Min}$. \\
\hline $\begin{array}{l}6 \mathrm{~h} . \\
6 \mathrm{~h} .\end{array}$ & $\begin{array}{l}33 \\
\text { Coff. natr. benz }\end{array}$ & 106 & 6 h. $0-10 \mathrm{m.}: 0,8$ \\
\hline $6 \mathrm{~h} .15 \mathrm{~m}$. & $34-32$ & 108 & 6 h. $10--20$ m.: 3,7 \\
\hline
\end{tabular}

$\mathrm{Zu}$ erwähnen wäre noch der Fall, dass nach Coffeïngabe der Blutdruck sinkt, trotzdem aber das Manometer nicht mit fällt. Hier ist durch die Diurese wenigstens das Absinken des Ureterendruckes verhindert worden.

Versuch III. Kaninehen, 1900 g. 0,8 pro $\mathrm{Kg}$ Paraldehyd. Blutdruck $110 \mathrm{~mm}$ Ureterendruck $40 \mathrm{~mm}$ Nach 0,05 Coff. natr. benz. $=81=040$

Es würde dieses in Parallele za setzen sein mit dem oben gesehilderten Versuch XII (s. o. S. 253), in welchem der Blutdruck durch Chloralhydrat herabgesetzt wurde, während dureh Kochsalzwassereinlauf Diurese bedingt und das Herabgehen des Ureterendruckes verzögert wurde, so dass Blutdruck und Ureterendruck sich immer mehr näherten und schliesslich so gut wie zusammenfielen, während noch Harn abfloss.

Es ist ersichtlich, dass es durch Einleiten von Diurese gelingt, den Ureterendruck auf Werthe zu treiben, welche die in der Norm beobachteten nicht unbeträchtlich ïbersteigen. So war der höchste erreichte Werth bei Kaninchen $84 \mathrm{~mm}$ (Coffeïndiurese), beim Hund $76 \mathrm{~mm}$ (Einlauf von 0,9 Proc. $\mathrm{NaCl}$ ). Starling hat (a. a. 0.) nach Diuretin beim Hund einen Ureterendruck von $92-94 \mathrm{~mm}$ erzielt.

Zweitens sieht man, dass die Steigerung des Ureterendruckes wohl bei der Diurese eintreten kann, und sehr oft in hohem Grade eintritt, aber durehaus keine regelmässige Begleiterseheinung der Diurese ist. Es giebt hochgradige Diuresen ohne Steigerung des. Ureterendruckes. 
258 XIV. Gottlieb und Magnus, Beziehungen des Ureferendruckes z. Diurese.

Was drittens die Abhängigkeit des Ureterendruckes vom Blutdruck betrifft, so wurde oben darauf hingewiesen, wie wechselnd die Differenz beider Grössen schon in der Norm sein kann. Diese - Unregelmässigkeit wird bei der Diurese noch vermehrt. Steigt der Ureterendruck bei gleichbleibendem Blutdruck, so muss ihre Differenz natürlich abnehmen. Es ist dieses wohl das häufigste Vorkommniss. Doch sind uns auch Fälle vorgekommen, in welchen die Differenz zunahm. Also auch hier ist eine regelmässige Beziehung nicht vorhanden.

Es ist wohl kein Zweifel, dass, wenn in unseren Versuchen Steigerung des Ureterendruckes eintritt, diese durch die Diurese bedingt ist. Die Art der Abhängigkeit ist aber noch unklar. Der Umstand, dass die Steigerung des Ureterendruckes bei der Diurese keine regelmässige und gesetzmässig auftretende Erscheinung ist, deutet auf complicirtere Beziehungen. Jedenfalls ist sicher, dass sie unabhängig von Aenderungen des Blutdruckes auftritt. Auch hieraus geht wieder hervor, dass es sehr unwahrscheinlich ist, dass der Ureterendruck in so einfacher Weise vom Blutdruck abhängen soll, wie dies Ludwig und Starling annehmen. Es mussen vielmehr noch andere Momente mitsprechen, und von den vorliegenden Hypothesen ist die einzige, welche mit unseren Beobachtungen im Einklange steht, die Heidenhain's, welcher annimmt, dass bei dem Druck des Ureterenmanometers sich Harnausscheidung und -Aufsangung das Gleichgewicht halten. Es ist danach der Stand des Ureterendruckes von zwei Variabeln abhängig. Daraus erklärt sich das verschiedene, scheinbar regellose Verhalten bei der Diurese.

$\mathrm{Ob}$ beim Zustandekommen des Ureterendruckes active Thätigkeit der secernirenden Elemente ( $\mathrm{Dr}$ eser) eine Rolle spielt, dafür geben unsere Versuche keine sicheren Beweise, wenn auch die Thatsache, dass Blutdruck und Ureterendruck fast vollständig einander genähert werden können, zu Gunsten dieser Annahme spricht. 